\title{
MRI-based heart and torso personalization for computer modeling and simulation of cardiac electrophysiology
}

\author{
Ernesto Zacur ${ }^{1}$, Ana Minchole ${ }^{2}$, Benjamin Villard ${ }^{1}$, Valentina Carapella ${ }^{4}$, \\ Rina Ariga ${ }^{3}$, Blanca Rodriguez ${ }^{2}$, Vicente $\mathrm{Grau}^{1}$ \\ ${ }^{1}$ Institute of Biomedical Engineering, Department of Engineering Science, \\ University of Oxford, UK \\ ${ }^{2}$ Department of Computer Science, University of Oxford, UK \\ ${ }^{3}$ Division of Cardiovascular Medicine, University of Oxford Centre for Clinical Magnetic \\ Resonance Research, UK \\ ${ }^{4}$ Simula Research Laboratory, Bærum, Norway
}

\begin{abstract}
In the last decade, electrophysiological models for in-silico simulations of cardiac electrophysiology have gained much attention in the research field. However, to translate them to clinical uses, the models need personalization based on recordings from the patient. In this work, we explore methodologies for the patient-specific personalization of torso and heart geometric models based on standard clinical cardiac magnetic resonance acquisitions to enable simulations. The inclusion of the torso and its internal structures allows simulations of the human ventricular electrophysiological activity from the ionic level to the body surface potentials and to the electrocardiogram.
\end{abstract}

\section{Introduction}

Biophysical electrophysiological (EP) models for the simulation of the electrical activity of the human heart are now approaching a mature state and are ready to be translated from an academic setting to medical research and clinical environments. Multiscale human ventricular EP models encapsulate mechanisms at the ionic and cellular level and incorporate a representation of tissue coupling to describe the propagation of the electrical excitation up to the whole organ (heart) electrical activity. In-silico simulations of virtual hearts present a research framework for the interpretation of medical data, allowing the assessment of biological hypothesis. In addition, they can be used to predict outcomes under simulated conditions such as cardiac or physiological dysfunctions, remodeling of the cardiac tissue, or drug treatments. Recently, EP models have moved from the study of general templates, either from a single subject or synthetic geometries, to be used with patient personalized data. This will be extended in the near future to play a role in the clinic to understand pathologies, stratify diseases, and also to optimize therapies. Several works have already made progress towards patient-specific personalized modeling, for example [1,2]. Structural and functional information of the heart is a key aspect in the personalization of the model. Patient-specific characteristics of the anatomy of the heart can be included into EP models by means of imaging studies including computer tomography (CT), magnetic resonance imaging, or ultrasound techniques. In particular, cardiac magnetic resonance (CMR) techniques provide a broad anatomical and functional 
information in a non-invasive manner and have become a routine diagnostic tool for some cardiovascular diseases. However, clinical CMR studies tend to consist of a sparse set of independent slices, which makes three-dimensional representation challenging.

Patient-specific electrical activities of the heart can be directly measured. Endocardial mappings record electrical signals by placing an electrode in different positions of the left endocardium using a catheter, and epicardial mappings get direct measures at the epicardial surface in an open chest surgery. Besides those invasive measures, body surface potential (BSP) mapping and the routinely used in the clinic electrocardiogram (ECG) measure the electrical activity of the heart non-invasively but in an indirect and global way.

In this work, we present a CMR based technique to geometrically personalize insilico human cardiac models from patient's CMR to enable forward EP simulations from the ionic level to the BSPs. The inclusion of the torso enables specifically the simulation of the electrocardiogram, which is the most widely used tool for evaluation of the human heart activity. It is worth mentioning that our proposed anatomical personalization could also be used in an inverse problem setting going from measured BSPs to epicardial activity. We will show that it is possible to accurately reconstruct the bi-ventricular and torso anatomy of the patient from a standard CMR acquisition protocol used in clinical practice with no more than localizers (scout images acquired at the beginning of the CMR session), 4-chamber and 2-chamber views and a stack of short axis slices from the base to the apex of the left ventricle in cine acquisition. Using standard CMR protocols is an important point since acquisition time in the clinic is a very limited and demanding resource and more extensive acquisition protocols are generally relegated to research. The focus on magnetic resonance studies is motivated by its noninvasiveness and also because of the ability of CMR studies to characterize multiple structural and functional parameters including scars, tissue or fiber microstructure, fat deposits, infarcted or fibrotic areas.

\section{Methods}

This section presents the details of our patient-specific reconstruction of a heart-torso anatomy derived from clinical CMR images with the final purpose to be used in an EP simulation framework up to the BSPs. Sample results for each step are shown together with the explanation of the methods.

\section{Bi-ventricular cardiac anatomy}

The cardiac tissue is segmented from long axis (LAX) views (4- and 2-chambers views) as well as from the stack of short axis (SAX) views of a cine acquisition. There are several software tools to perform tissue segmentation, including commercial products widely used in the clinical practice (see [3] for references) as well as other research tools (for example, [4]). These offer different degrees of automation, from complete manual contouring, semiautomatic methods to reduce the operator time, up to fully automated tools. Which of them is the best option for cardiac images is debatable, on the one hand, manual contouring is usually considered as a ground truth, while automatic methods avoid operator bias, unintentional errors and have 
repeatability. In this work, we use manual contours from a well-trained expert with several years of experience. The contouring follows the general guidelines and consensus recommendations from [5]. Contouring includes left epicardium (including the septum), left endocardium (excluding papillary muscles), and right endocardium from and to the septum (excluding trabeculations). Exemplary LAX and SAX contours are shown in Fig. 1. Images in the example correspond to the end diastolic (ED) frame.

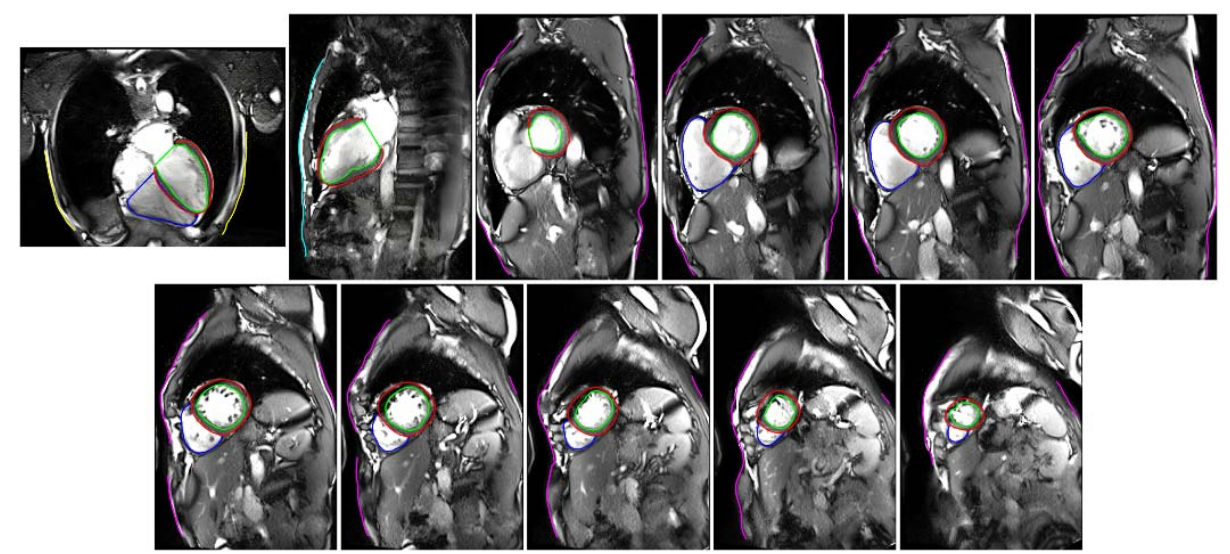

Fig. 1. Heart cine slices and their contours at ED. From left to right and from top to bottom: 4- and 2chamber views, short axis stack from the most basal to the most apical slice. Left epicardium contours in red, left endocardium contours in green, and right endocardium contours in blue.

Since we are interested in bi-ventricular geometry, the right ventricle endocardium borders are needed. However, the image resolution does not usually allow an accurate delineation. Therefore, we synthesize the right epicardium contour as an offset of the right endocardium at a distance of $4 \mathrm{~mm}$ [6]. After that, contours are automatically reorganized into a complete epicardial contour (EPI), the left endocardium (LV), and the right endocardium (RV), now including the septum (see Fig. 2).
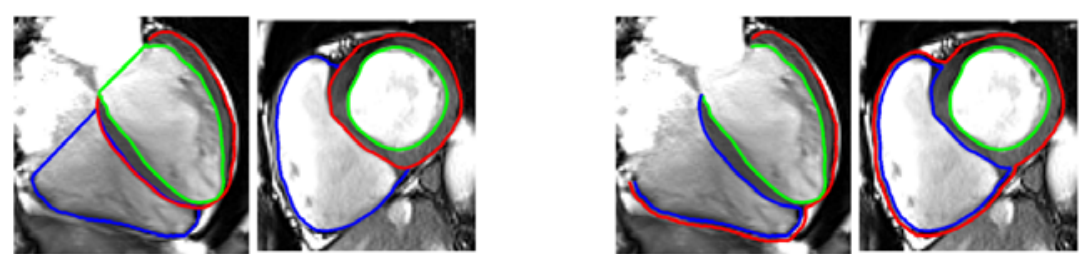

Fig. 2. Re-organization of the contours: synthesis of the right epicardium as part of the whole epicardium and split the left ventricle into epicardium and septum.

Due to different cine slices being acquired at different breath holds, misalignments and spatial inconsistencies usually appear among the contours. We use the methodology described in [7] to correct for these misalignments. In summary, the alignment method computes the optimal rigid transformation in 3D for each slice for the contours to define spatially consistent surfaces. Realigned contours are shown in Fig. 3. 

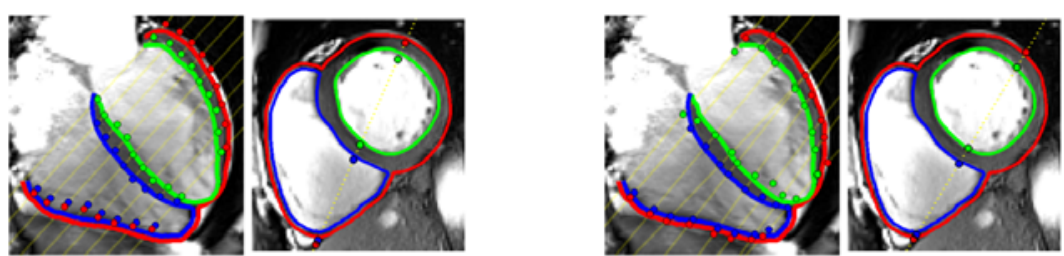

Fig. 3. Alignment of the slices to correct the breath motion. The spatial consistency of the contours is improved as can be seen in the lateral wall of the right ventricle. Left: discrepancies between contours from intersecting slices before correction. Right: after correction the contours intersecting contours are more consistent.

Once the contours are consistently aligned, a surface is interpolated for each of the structures (EPI, LV, and RV), following [8]. As in the case of the correction for alignment of the slices, the use of LAX views is key at this step. In particular, the apical area can only be well resolved using LAXs. The three surfaces are clipped at the level of the most basal SAX and they build the bi-ventricular heart geometry. Reconstructed surfaces together with the contours are shown in Fig. 4.
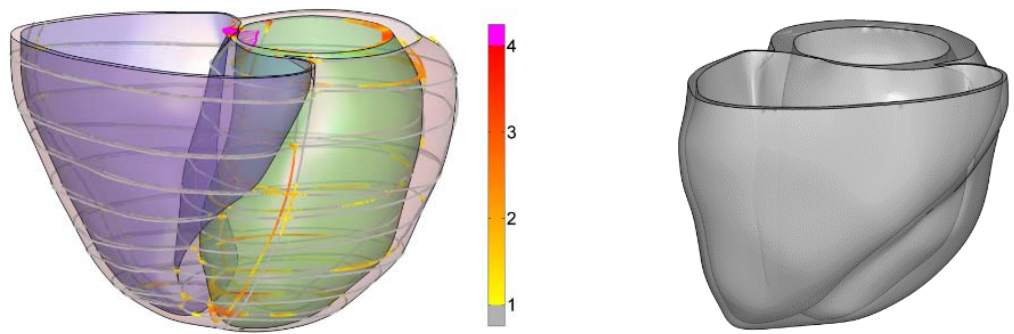

Fig. 4. Left: contours and the reconstructed surfaces of the heart. Each contour point is colored according to its distance to the corresponding surface (distances in $\mathrm{mm}$ ). Right: another view of the final bi-ventricular heart.

\section{Body and torso anatomy}

Unlike CT, cine CMR does not usually produce a volumetric image but a sparse set of 3D oriented slices. Therefore classical surface generation techniques from volumetric data such as marching cubes cannot be used. This makes the reconstruction of the body surface (BS) challenging since only very sparse information of the torso is available. Some previous approaches have dealt with this scenario to reconstruct the body: [9] proposes a human model customization from monocular photos; [10] uses a manual affine deformation of a template to sagittal, coronal and axial MR images; [11] combined CT and MRI scans to make a tetrahedral mesh of a pig thorax; and [12] also uses MRI and CT scans to personalize a human geometry. In this work, we propose to fit a statistical shape model (SSM) of human bodies to the BS contours that we can obtain from the MR images. Although the CMR images are not acquired with a focus on body surface extraction, their field of view normally includes parts of the BS. Besides, at the start of the CMR studies, scout images in sagittal, coronal and axial views are commonly acquired to be used as localizers. We contoured the BS from all these images, using a semiautomatic tool that extracts isophote curves in the clearly contrasted interface between air and skin. The extracted contours are shown in 
Fig. 5, where the sparsity of the data can be appreciated as well as the presence of large regions (such as the upper-right part of the chest) with no information at all.
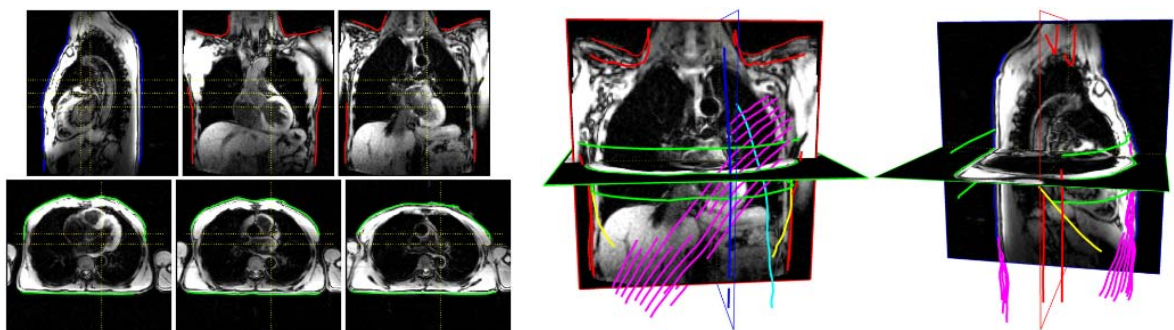

Fig. 5. Left: BS contours on the localizers. Right: 3D arrangement of all the BS controls, including the ones that can be extracted from heart focused slices (see Fig. 1).

A SSM of body shapes ${ }^{1}$ built from 4300 subjects [13] is fitted to the contours. A rigid transformation together with the first 40 principal components of the SSM were considered in the fitting. As initial condition, the mean shape is situated in accordance with the previously reconstructed heart. The fitting is performed by a gradient descent to minimize the root mean square (RMS) contours-to-surface distance. Figure 6 shows an example of the fitting with a final RMS of $6.7 \mathrm{~mm}$. After the SSM fitting, a final deformation is performed via approximate thin plate splines (TPS) $[14,15]$ to improve the agreement with the contours, resulting in a final RMS of $3.4 \mathrm{~mm}$.
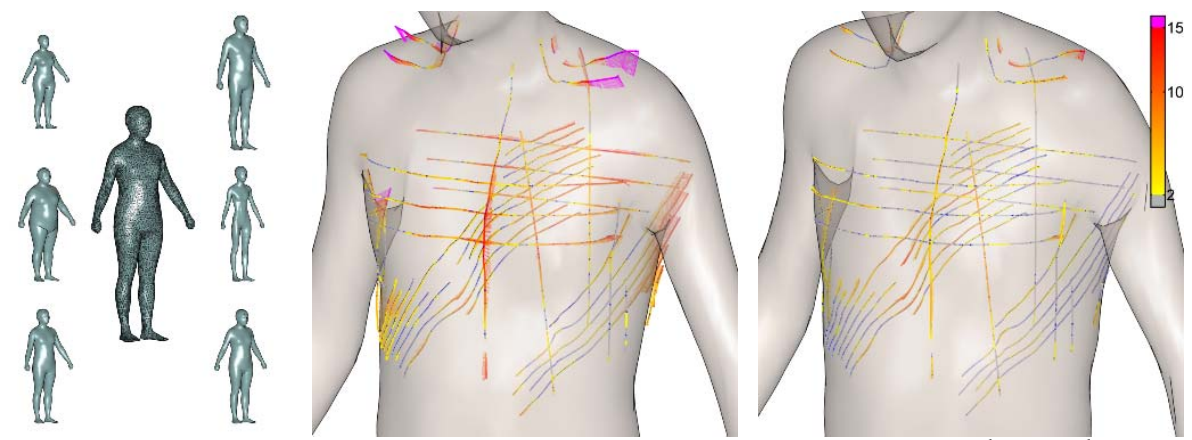

Fig. 6. Left: mean shape of the body SSM with its extreme shapes along the $1^{\text {st }}, 2^{\text {nd }}$, and $3^{\text {rd }}$ principal modes. Center: the result of the SSM fitted to the body contours. Points of the contours are colored according to their distances to the surface. Right: the result of TPS deformation towards the contour points to reduce the contour-to-surface distances.

\section{Ribs and lungs}

In order to compute BSPs, the electrical potentials at the heart surface have to be propagated to the skin. In that path, the impedances of the different tissues between the epicardium and the skin have to be considered. The inclusion of the different organs for computational studies is controversial with some works reporting minor contributions of different organ impedances on BSP [16] and others reporting significant effects due to uncertainty in impedances [17]. Therefore, for potential sensitivity

1 http://humanshape.mpi-inf.mpg.de/ 
analysis, we include ribs and lungs within the torso representation. These internal structures are incorporated through template deformation. As we represent the BS by a deformed mean SSM mesh with fixed anatomical point correspondences, a representation of ribs and lung in that mean SSM mesh coordinates system is sufficient. This template was built from the segmentation of a CT acquisition of a single subject, for which its BS was deformed to the mean SSM shape. The deformation of the internal structures was smoothly extrapolated by means of TPS. Figure 7 shows the ribs and lungs template with correspondences at the BS level with the SSM model. This template is finally deformed to the personalized torso, extrapolating the deformation of the correspondent anatomical points by TPS.
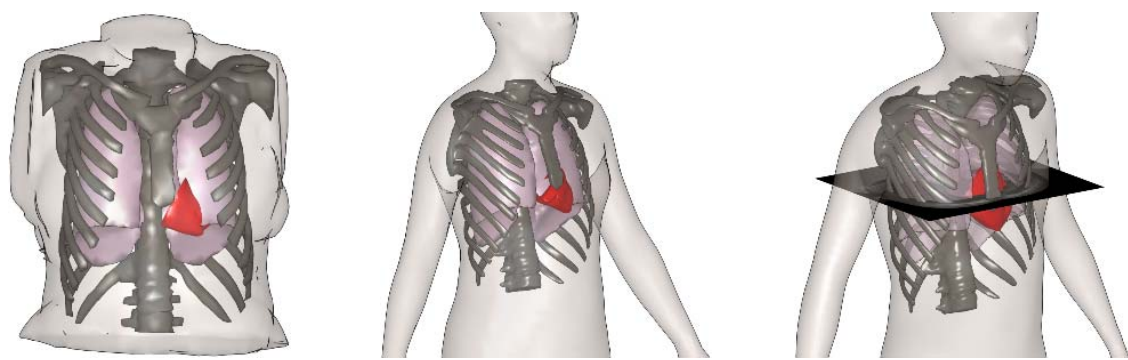

Fig. 7. Left: segmented internal structures of the torso obtained from a CT of a single subject. Center: transfer of the internal structures to the mean SSM shape. Left: deformation from the mean SSM to the patient personalized body shape (only ribs and lungs are transferred to the patient geometry whilst the heart is the one reconstructed from the patient images).

\section{Remeshing final surfaces and volumetric meshes}

The majority of biophysical EP models are solved using the finite element method (FEM) with unstructured meshes of tetrahedral elements. The meshes allow the tessellation of complex geometric objects and the specification of different properties for each element depending on which structure it belongs to. In our case of a full body simulation, different spatial scales have to be considered. Spatial resolutions smaller than $0.5 \mathrm{~mm}$ are required to resolve the propagation of the electrical activation within the myocardium with acceptable accuracy. For example, a spatial resolution of 0.4 $\mathrm{mm}$ has been used in [18] to get an error in the conduction velocity below $10 \%$. However, due to the different nature of the physical mechanism, larger spatial resolutions can be used to solve the propagation of the epicardial potentials to the BS. The heart surface (Fig. 4 right) is remeshed with a restricted Frontal-Delaunay algorithm using the mesh generator JIGSAW $^{2}$ [19] with a specified element size of $0.4 \mathrm{~mm}$. The other structures are remeshed with the same software with the following element sizes: 2 $\mathrm{mm}$ for the ribs; $3 \mathrm{~mm}$ for the lungs; and $10 \mathrm{~mm}$ for the torso. Once surfaces have been remeshed, TETGEN ${ }^{3}$ [20] is used to build the final tetrahedral mesh (see Fig. 9 left). The resulting tetrahedral meshes have a number of elements in the order of $40 \times 10^{6}\left(7 \times 10^{6}\right.$ nodes), of which $30 \times 10^{6}\left(5 \times 10^{6}\right.$ nodes) correspond to the myocardium.

2 http://github.com/dengwirda/jigsaw

3 http://wias-berlin.de/software/tetgen/ 


\section{Results}

Figure 8 shows the acquired slices together with their intersection with the BS, ribs, and lungs for a visual assessment of the fitting of the torso and the placement of the internal structures. A quantitative assessment was performed in terms of the distances from the manual contour points to the reconstructed meshes. For the epicardium surface, the resulting RMS of contours-to-mesh distance is $0.5 \mathrm{~mm}$ with $90 \%$ of the points with a distance below to $0.75 \mathrm{~mm}$. For the left endocardium, RMS is $1.35 \mathrm{~mm}$ and $90 \%$ percentile $2.2 \mathrm{~mm}$. For the right endocardium, RMS is $0.45 \mathrm{~mm}$ and $90 \%$ percentile $0.72 \mathrm{~mm}$. Finally, to evaluate the reconstruction of the body surface, RMS is $3.44 \mathrm{~mm}$ and $90 \%$ percentile $5.5 \mathrm{~mm}$.

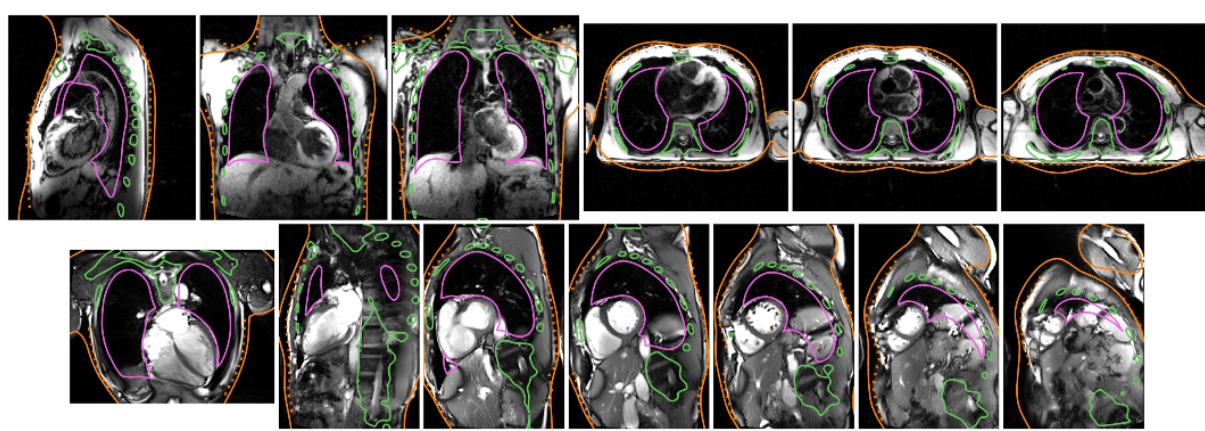

Fig. 8. Visual assessment of the fitted torso and the reconstructed internal surfaces. Dotted lines correspond to the manual delineations of the BS and continuous lines correspond to the intersection of the image plane with reconstructed the surfaces.

A bi-domain EP simulation following the O'Hara-Rudy model was conducted in $\mathrm{CHASTE}^{4}$ [21] using the patient-specific personalized mesh. Early activation sites in the endocardium were selected in accordance with physiological knowledge [22]. Others physiological parameters were set to standard values found in the literature. Fiber microstructure was built following the Streeter rule from -60 degrees helix angle at endocardial surfaces to +60 degrees at epicardium [23]. BSPs were computed by propagating the electrical activity from the epicardium. Figure 9 depicts the obtained BSPs. In addition, we can virtually place electrodes on the limbs and the surface chest to synthesize a 12-lead ECG.

4 http://www.cs.ox.ac.uk/chaste/ 

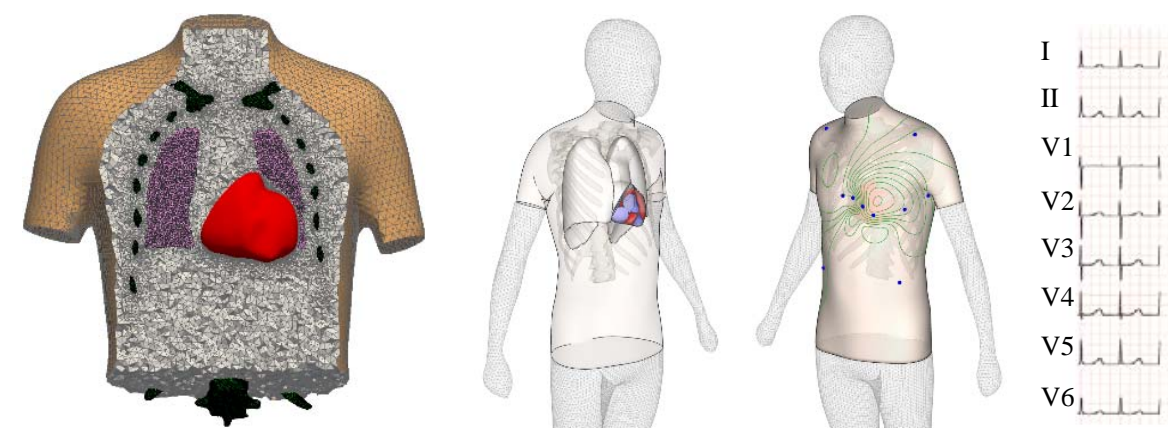

Fig. 9. From left to right: final tetrahedral mesh for FEM simulations (each internal structure having a label and specific properties); epicardial potentials (in arbitrary units) during the activation; isolevels of the BSPs and positions of the virtual electrodes (blue dots); synthesized ECG.

\section{Conclusion}

We have described the components of a pipeline for the reconstruction of accurate patient-specific personalized models of heart and torso from CMR data. We show that model personalization can be performed using classical CMR protocols, with no need of extra acquisitions. The reconstruction of the torso geometry, with the accurate localization of the heart, allows the EP models to simulate the cardiac electrical activity from ionic currents up to the BS and the ECG. The inclusion of other structural and functional information from other CMR modalities such as scars or fibrotic areas can be easily performed. BSPs can be used to synthesize ECG signals that can be compared with the patient ECG. This pushes the use of in-silico EP models towards the clinical practice with several applications such as the understanding of pathologies, risk stratification, optimization and following of therapies.

Acknowledgments. EZ acknowledges the Marie Sklodowska-Curie Individual Fellowship from the H2020 EU Framework Programme for Research and Innovation (Proposal No: 655020-DTI4micro-MSCA-IF-EF-ST). AM and BR are supported by BR's Wellcome Trust Senior Research Fellowship in Basic Biomedical Sciences, the CompBiomed project (grant agreement No 675451) and the NC3R Infrastructure for Impact award (NC/P001076/1). BV acknowledges the support of the RCUK Digital Economy Programme grant number EP/G036861/1 (Oxford Centre for Doctoral Training in Healthcare Innovation). VC was supported by ERACoSysMed through a grant to the project SysAFib - Systems medicine for diagnosis and stratification of atrial fibrillation. RA is supported by a British Heart Foundation Clinical Research Training Fellowship. VG is supported by a BBSRC grant (BB/I012117/1), an EPSRC grant (EP/J013250/1), by BHF New Horizon Grant NH/13/30238 and by the CompBiomed project (grant agreement No 675451).

\section{References}

1. H. Arevalo, et al. "Arrhythmia risk stratification of patients after myocardial infarction using personalized heart models.” Nature Communications, 7, 2016.

2. O. Zettinig, et al. "From medical images to fast computational models of heart electromechanics: An integrated framework towards clinical use.” In Proceedings FIMH 2013.

3. P. Peng, et al. "A review of heart chamber segmentation for structural and functional analysis using cardiac MRI.” Magn Reson Mater Phy, 29(2):155-195, 2016. 
4. E. Heiberg, et al. "Design and validation of segment - freely available software for cardiovascular image analysis.” BMC Medical Imaging, 10(1):1, 2010.

5. J. Schulz-Menger, et al. "Standardized image interpretation and post processing in cardiovascular magnetic resonance: Society for cardiovascular magnetic resonance (SCMR) board of trustees task force on standardized post processing.” J Cardiovasc Magn Reson, 15(1):35, 2013.

6. R. Prakash. "Determination of right ventricular wall thickness in systole and diastole. Echocardiographic and necropsy correlation in 32 patients.” Heart, 40(11):1257-1261, 1978.

7. B. Villard, et al. "Correction of Slice Misalignment in Multi-breath-hold Cardiac MRI Scans.” In Proceedings STACOM 2016.

8. B. Villard, et al. "Cardiac Mesh Reconstruction from Sparse, Heterogeneous Contours.” In Proceedings MIUA 2017.

9. S. Zhu, et al. "An efficient human model customization method based on orthogonal view monocular photos.” Comput Aided Des, 45(11):1314-1332, 2013.

10. O. Zettinig, et al. "Data-driven estimation of cardiac electrical diffusivity from 12-lead ECG signals.” Med Image Anal, 18(8):1361-1376, 2014.

11. K. Gillette, et al. "Generation of combined-modality tetrahedral meshes.” In Proceedings CinC 2015.

12. N. Trayanova, et al. "How computer simulations of the human heart can improve antiarrhythmia therapy.” J Physiol, 594(9):2483-2502, 2016.

13. L. Pishchulin, et al. "Building statistical shape spaces for 3D human modeling." Pattern Recogn, 67:276-286, 2017.

14. K. Rohr, et al. "Landmark-based elastic registration using approximating thin-plate splines.” IEEE Trans Med Imaging, 20(6):526-534, 2001.

15. B. Amberg, et al. "Optimal step nonrigid ICP algorithms for surface registration.” In Proceedings IEEE CVPR 2007.

16. S. Geneser, et al. "Application of stochastic FEM to study the sensitivity of ECG forward modeling to organ conductivity.” IEEE Trans Biomed Eng, 55(1):31-40, 2008.

17. D. Keller, et al. Ranking the influence of tissue conductivities on forward-calculated ECGs, IEEE Trans Biomed Eng, 57(7):1568-1576, 2010.

18. M. Bernabeu, et al. "Shock-induced arrhythmogenesis in the human heart: A computational modelling study.” In Proceedings IEEE EMBS 2010.

19. D. Engwirda. "Locally-optimal Delaunay-refinement and optimisation-based mesh generation.” Ph.D. thesis, The University of Sydney, 2014.

20. Hang S. "TetGen, a Delaunay-based quality tetrahedral mesh generator." ACM Trans Math Softw, 41(2):11:1-11:36, 2015.

21. J. Pitt-Francis, et al. "Chaste: A test-driven approach to software development for biological modelling.” Comput Phys Commun, 180(12):2452-2471, 2009.

22. L. Cardone-Noott, et al. "Human ventricular activation sequence and the simulation of the electrocardiographic QRS complex and its variability in healthy and intraventricular block conditions.” EP Europace, 18(suppl4), 2016.

23. D. Streeter "Gross Morphology and Fiber Geometry of the Heart." Johns Hopkins Press, 1979. 